\title{
ELECTRONIC DIPOLE RESONANCE IN SMOKY QUARTZ
}

\author{
J. KERSSEN and J. VOLGER \\ Fysisch Laboratorium der Rijksuniversiteit, Utrecht. \\ The Netherlands
}

Received 28 April 1967

\begin{abstract}
Microwave absorption in smoky quartz mono-crystal is ascribed to resonance transitions of trapped electrons between initially configurational degenerated states, which are Stark-splitted by a polarizing electric field.
\end{abstract}

In special cases a centre in a solid at which a hole or an electron has been trapped, may act as an electric dipole that will exhibit a specific moment. In the dielectric constant of the solid a Langevin-type of contribution may then be found and Debye-type dielectric relaxation losses may occur which are determined by a temperature dependent relaxation time governing the dynamics of the dipole orientation [1-5]. The wave function of the trapped electron may be thought to be a linear combination of atomic wave functions, centred at certain equivalent lattice sites. When these atomic wave functions have only little overlap, the configurational degeneracy is hardly removed in the absence of a polarizing field. Such a field, however, will easily cause a large linear Stark-splitting of the multiplet and will localize

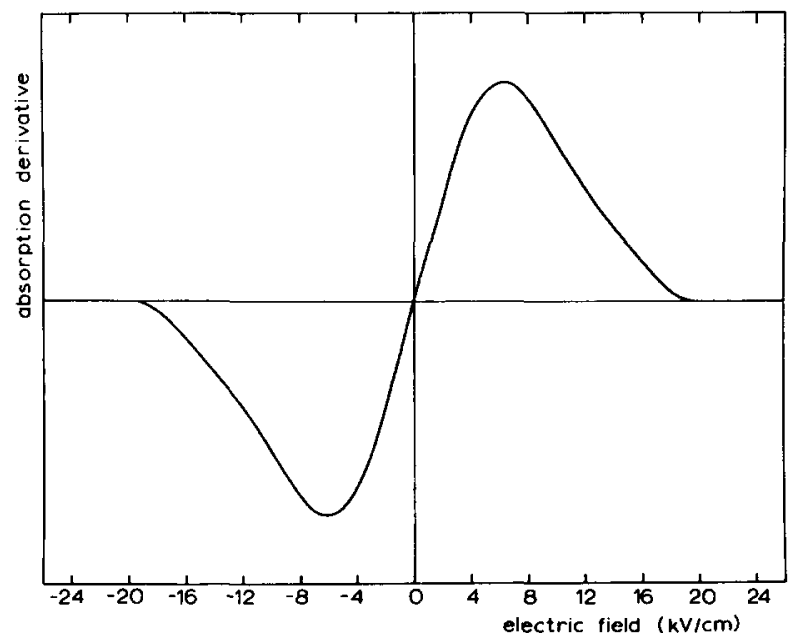

Fig. 1. Electronic dipole resonance absorption derivative spectrum of the colour centres in smoky quartz crystal at $4.2^{\circ} \mathrm{K}$ and $9.5 \mathrm{GHz}$ with the electric fields parallel to the crystal $\mathrm{c}$-axis. the electrons according to Boltzmann-statistics. Transitions between these localized, asymmetrized states are thermally induced, causing the dielectric relaxation mentioned. Moreover, the distance between the Stark-levels may be easily such, that resonance with a microwave field should be possible also. Such an effect. which we suggest to give the name of electronic dipole resonance has been predicted [6] and is the subject of the present note. After various unsuccessful efforts we have been able to detect what is probably the first case of electronic dipole resonance in solids, though our observations may be largely influenced by strong dipole-dipole interaction.

The centres investigated are colour centres in smoky quartz, which have been studied extensively in various respects $[7,8]$. The dipole strength is about 4 Debye units [4] $\left(=4 \times 10^{-18}\right.$ e.s.e. $)$. Therefore very roughly estimated resonance transitions should occur at field strengths at the order of $2 \mathrm{kV} / \mathrm{cm}$ when an excitation frequency of $10 \mathrm{GHz}$ is used.

Recently various groups [12-17] investigated the ionic dipole resonance of centres in $\mathrm{KCl}$, with experimental techniques that slightly differed from ours. We designed a re-entrant type microwave cavity, basically a piece of coaxial waveguide. short circuited at two sides and with a small gap in the middle of the inner conductor. With this cavity it is possible to submit samples at the same time to an electric r.f. field parallel to a static electric field. The whole cavity can be immersed in liquid helium. At $4.2^{\circ} \mathrm{K}$ the $Q$ is about 5000 . For the detection of absorption an ordinary homodyne $3 \mathrm{~cm}$ spectrometer was used. Various samples were investigated. The best results have so far been obtained with a slice cut perpendicular to the crystal c-axis out of a natural smoky quartz monocrystal, homogeneous and only slightly con- 

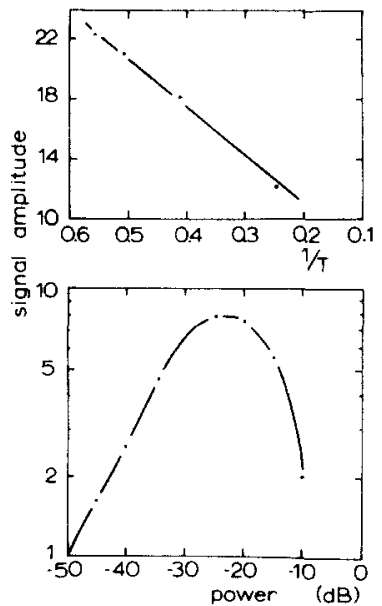

Fig. 2. Signal amplitude as a function of the microwave energy incident on the resonant cavity. $0 \mathrm{~dB}$ corresponds to an incident power of about $0.05 \mathrm{~W}$. Tentative measurements of the dependence of temperature are given in the upper part of the graph for the field strength that gives maximum absorption derivative.

tamined with aluminium (about $7 \mathrm{ppm}$ ). The electric fields were applied parallel to the c-axis. No resonances were found at $77^{\circ} \mathrm{K}$, the relaxation time being probably too short then and the energy levels too much disturbed by a phonon interaction. At liquid helium temperatures we observed a rather strong response with the character of a very broad absorption line centred at a field strength of about zero, as drawn in fig. 1. Variation of the thickness of the quartz samples and of the width of the cavity-gap showed that the signal is a unique function of the static field strength. Investigation of the power dependence of the line revealed a saturation type behaviour. At low power level the signal amplitude is proportional to the square root of the power incident on the cavity. while at higher power levels it sharply decreases (see fig. 2) when measured at $4.2^{\circ} \mathrm{K}$. Tentative measurements of the temperature dependence of the absorption show a $1 / T$-relation of the amplitude (fig. 2). The saturation behaviour is rather independent of $T$ down to about $2^{\circ} \mathrm{K}$.

Removing the colour centres by heating the quartz at $600^{\circ} \mathrm{K}$ removes also the absorption signal. while $\mathrm{X}$-irradiation brings back both signal and colour centres. Measurements of the angular dependence of the signal are to be carried out yet.

In explaining the observed microwave absorption. use can be made of some exising model theories and, moreover, the analogy with the case of ionic dipole resonance may be remarked [9-11]. However, there is one feature that calls for atten- tion, viz. the very strong coupling of the centre with the lattice, which makes the positioning of the energy levels a much less clear-cut affair than in the case of ESR or, perhaps, even in the case of ionic dipole resonance. The potential wells at the relevant sites that provide the atomic wave functions mentioned above, are strongly influenced by local distortion owing to lattice oscillations and strain. Moreover, large "internal fields" may exist, due to dipole-dipole interaction, in our case perhaps $1 \mathrm{kV} / \mathrm{cm}$. The energy levels between which the resonant microwave field absorption takes place are therefore to be considered as bands with some width, possibly one order of magnitude larger than the microwave phonon energy. This explains why zero field absorption occurs and the absorption line is widened so much. One could consider the effect primarily as phonon production by microwave conversion at the centres. However, the resonant character of the phenomenon appears clear from the observation that at sufficiently high polarizing field the absorption decays sharply.

Under these circumstances it is hard to extract detailed information from the electronic dipole resonance spectrum observed and to derive the parameters of the problem's Hamiltonian.

The authors gratefully acknowledge the hospitality of Philips Research Laboratories, Eindhoven. the Netherlands, where measurements at liquid helium temperatures could be carried out, and the help of M. M. Gerritsen and van der Boom.

1. J. Volger, Disc. Faraday Soc. 23 (1957) 63.

2. J. Volger, Progress in Semiconductors 4 (1959) 205.

3. J. M. Stevels and J. Volger, Philips Res. Repts. 17 (1962) 283.

4. W. J. de Vos and J.Volger, Physica, to be published.

5. W. J. de Vos and J.Volger, Phys. Letters, 24A (1967) 539

6. J. Volger, Inaugural Address, Utrecht, 1959.

7. M. C. M. O'Brien, Proc. Roy.Soc. (London) Ser. A 231 (1955) 404.

8. A. Kats, Philips Res. Repts. 17 (1962) 133.

9. H. B. Shore, Phys. Rev. 151 (1966) 570.

10. P.Sauer, O.Schirmer and J.Schneider, Phys. Stat. Sol. 16 (1966) 79.

11. J.A.Sussmann, Phys. kondens. Materie 2 (1964) 146; J. Chem. Phys., to be published.

12. U. Kuhn and F. Lutty, Solid State Comm. 4 (1965) 31.

13. W. E. Bron and R.W. Dreyfus, Phys. Rev. Letters 16 (1966) 165.

14. G. Feher, I. W. Shepherd and H. B. Shore, Phys. Rev. Letters 16 (1966) 500 .

15. L.D. Schearer and T.L. Estle, Solid State Commun. 4 (1966) 639.

16. T. L. Estle, private communication.

17. G. Höcherl, D. Blumenstock and H. C. Wolf, Phys. Letters, 24A (1967) 511, and private communication. 